\title{
Gérer les temps pour gérer la qualité : l'activité de traitement des dossiers de retraite
}

Manage Time to Manage Quality: The Activity of Retirement Case File Processing

Valérie Zara-Meylan et Serge Volkoff

\section{(2) OpenEdition}

Journals

Édition électronique

URL : http://journals.openedition.org/activites/4769

DOI : 10.4000/activites.4769

ISSN : $1765-2723$

Éditeur

ARPACT - Association Recherches et Pratiques sur les ACTivités

Référence électronique

Valérie Zara-Meylan et Serge Volkoff, « Gérer les temps pour gérer la qualité : l'activité de traitement des dossiers de retraite », Activités [En ligne], 16-2 | 2019, mis en ligne le 15 octobre 2019, consulté le 17 octobre 2019. URL : http://journals.openedition.org/activites/4769; DOI : 10.4000/activites.4769

Ce document a été généré automatiquement le 17 octobre 2019.

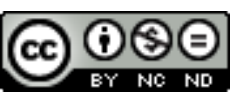

Activités est mis à disposition selon les termes de la licence Creative Commons Attribution - Pas d'Utilisation Commerciale - Pas de Modification 4.0 International. 


\section{Gérer les temps pour gérer la qualité : l'activité de traitement des dossiers de retraite}

Manage Time to Manage Quality: The Activity of Retirement Case File Processing Valérie Zara-Meylan et Serge Volkoff

\section{NOTE DE L'ÉDITEUR}

Article soumis le 13 juin 2018, accepté le 25 mars 2019

Les auteurs remercient vivement: Céline Mardon et Corinne Gaudart, pour leur participation à cette recherche; les techniciens retraite, leurs responsables, et les membres du groupe de pilotage (en particulier Mmes Charles-Bray, Géhin, Maillebuau et M. Grosse) pour le temps qu'ils ont consacré à cette étude et leurs précieux commentaires.

La recherche d'où est issue cette publication a bénéficié d'un financement de la Cnav dans le cadre d'une convention de recherche avec le GIS-Creapt.

1 Cet article porte sur la construction de la qualité par la gestion temporelle dans une activité associant traitement de dossiers et prestation de service. Il s'appuie sur une recherche qui examine les modalités de gestion des multiples cadres temporels de leur activité par des techniciens qui ont un rôle clé pour la qualité de la prestation fournie par un organisme administratif.

2 Son point de vue est d'aborder les liens entre gestion temporelle et gestion de la qualité en considérant l'enrichissement mutuel de ces dimensions d'analyse.

3 Les recherches en ergonomie ont montré depuis longtemps l'ancrage de l'activité dans le temps et dans la dynamique des situations qu'elle-même participe à produire (Teiger, 1987). C'est particulièrement le cas lorsque les travailleurs ont à gérer de multiples conditions temporelles pour influer sur le déroulement des processus conduits, et aussi pour faire évoluer ces conditions temporelles elles-mêmes (Cellier, De Keyser, \& Valot, 
1996). Pour cela, les règles, procédures, objectifs quantifiés, articulations et interrelations prescrites, etc., qui imposent un ensemble de contraintes, constituent aussi potentiellement des ressources pour la gestion temporelle dans l'activité. Ces conditions du travail fournissent ainsi plus ou moins de possibilités pour agir, et elles façonnent la structuration située de l'action (Norros, 2004).

4 Ces propriétés peuvent être transposées aux « cadres temporels », un concept que nous avons emprunté à W. Grossin (1996) pour identifier les différentes constructions temporelles rigides, aux assemblages divers et hétérogènes, issues des conditions du travail (Zara-Meylan, 2016); ces cadres temporels sont porteurs d'exigences qui délimitent, contraignent, mais aussi structurent et soutiennent l'activité.

Élucider les dimensions de « la qualité » dans l'activité fournit un fil conducteur pour spécifier la diversité et les caractéristiques de ces cadres temporels et de leurs configurations, et pour comprendre les modalités par lesquelles chacun gère son milieu temporel en visant cette qualité. En sens inverse, l'analyse des cadres temporels, de leurs imbrications, des stratégies qu'adoptent les travailleurs pour gérer leurs configurations évolutives, des obstacles qui fragilisent cette gestion et des ressources qu'ils élaborent avec l'expérience pour la consolider, permet de jeter un autre regard sur la qualité et d'en déplier les composantes.

6 C'est l'approche qui a été menée auprès de techniciens en charge de dossiers retraite et de l'accompagnement des assurés, à partir d'une demande de responsables de la Caisse Nationale d'Assurance Vieillesse (CNAV). Cette demande, formulée durant une période (2013-2015) marquée par d'importants changements techniques et organisationnels (Zara-Meylan \& Volkoff, 2017), portait sur la justesse des traitements de dossiers de retraite, avec en particulier une préoccupation concernant la fréquence des " erreurs » telle que relevée par les indicateurs. Une question sous-jacente était de savoir à quelles conditions les changements en cours allaient permettre de progresser en ce domaine, ou provoqueraient au contraire de nouvelles difficultés. S'ajoutait à cela - en raison de réformes des retraites qui contribuent à élever les âges de départs, y compris ceux des personnels de la Branche Retraite eux-mêmes - une inquiétude quant à l'adaptation des techniciens les plus anciens à ces changements qui transforment leurs repères, voire leurs métiers.

7 L'étude a été menée dans quatre agences et a porté sur trois métiers, centraux dans la production des prestations retraite : d'abord les techniciens Conseil Retraite chargés de la liquidation des dossiers, vers lesquels était plus particulièrement orientée la demande initiale ; mais aussi les techniciens Conseil Carrières qui assurent en amont la reconstitution des carrières, et les techniciens Conseil Contrôleurs qui, en aval, vérifient la conformité des dossiers constitués.

8 Notre propos dans cet article va consister d'abord à rendre davantage visibles les enjeux de qualité et de gestion des temps, par un examen d'ensemble des conditions et contraintes des situations dynamiques à gérer par ces techniciens [1]. Nous indiquerons ensuite les connaissances et modèles d'analyse mobilisés dans notre approche des cadres temporels et de la gestion du milieu temporel par les techniciens [2], puis la démarche méthodologique dans cette perspective [3]. Les résultats présentés ensuite [4] viseront à analyser la gestion de ce "milieu», d'abord dans des configurations de l'activité quotidienne [4-1], puis dans des configurations de régularisation des relevés de carrière [4-2], d'installation des rendez-vous avec les assurés [4-3], et de gestion des «erreurs » retournées par le Contrôle [4-4]. Nous 
conclurons [5] en indiquant quelques pistes de réflexion et d'action que ces analyses auront permis d'ouvrir.

\section{Les enjeux de qualité pour les techniciens, dans une activité en situation dynamique}

La finalité du métier des techniciens Conseil, rappelée dans les fiches métier internes à l'organisme, est de "permettre l'évaluation et l'attribution des prestations retraite aux assurés, en fonction de leur situation ». Ils sont pour cela chargés de constituer les dossiers des assurés, selon la règlementation en vigueur. En articulation avec les autres services d'accueil et de conseil de la Branche, leur mission est aussi d'informer les assurés, les conseiller dans leurs choix, les orienter éventuellement auprès de partenaires de la protection sociale et les suivre dans leurs démarches en contactant d'autres organismes si nécessaire.

L'activité des techniciens ne doit pas être seulement considérée dans l'immédiateté du traitement des dossiers, mais dans des situations dynamiques aux contraintes évolutives (Cellier et al., op. cit.). Les dossiers des assurés s'apparentent à des processus multiples et longs, à suivre sur trois à quatre mois, parfois davantage. Leurs stades d'avancement sont divers et chacun évolue en permanence dans le portefeuille d'un technicien, non seulement au fil de son travail articulant back-office et front office (Denis, 2011 ; Petit, 2005), mais aussi au fil du temps, à mesure qu'une date d'effet ${ }^{1}$ ou une butée correspondant à un délai prédéterminé se rapprochent. En outre, des collègues ou des organismes extérieurs peuvent adresser des éléments complémentaires ou au contraire tarder à les envoyer. Enfin l'assuré lui-même peut effectuer des demandes, intervenir dans l'avancée de son dossier ou au contraire rester longtemps silencieux alors même qu'une réaction de sa part serait attendue.

11 Ces processus multiformes sont cadrés dans l'organisme par des systèmes de prescription et d'évaluation eux-mêmes évolutifs. Ils sont suivis par des indicateurs de gestion régionaux et nationaux qui concernent le nombre de dossiers traités ainsi que la qualité de la liquidation, avec en particulier la justesse et la complétude des dossiers et les délais de traitement. La qualité de la prestation est aussi estimée à partir de la "satisfaction de l'assuré ", un indicateur appuyé sur des enquêtes régulières qui a été déployé dans le mouvement des indicateurs de gestion. Le service de proximité en agence reste essentiel dans cette perspective, mais la tendance actuelle est à la suppression de "l'accueil spontané » sans rendez-vous préalable, ce qui cadre davantage et souvent restreint aussi les moments d'échanges avec l'assuré.

De ce tour d'horizon du contexte et des premiers échanges dans les agences, est ressorti l'intérêt de déplacer la question des taux d'erreurs, en montrant plus largement en quoi, tout au long du processus de traitement des dossiers et de liquidation, l'activité des techniciens consiste à fiabiliser la prestation retraite dans un ensemble de plusieurs dimensions : la justesse en fonction de la situation de chaque assuré, de la législation et des procédures, certes, mais aussi les délais de traitement conformément aux règles nationales et locales; ce à quoi s'ajoutent une visée de clarté pour l'assuré qui, au-delà d'une notion de "satisfaction ", intègre plutôt une dimension de compréhension et de choix en connaissance de cause, et enfin une dimension de précision des traces laissées 
pour soi et pour les autres, dans le système informatique et dans les dossiers, autres documents ou notes.

Ces quatre objectifs constituent pour nous les composantes de la «qualité » telle qu'on l'aborde dans cette recherche. Dans leur principe ils ont une cohérence d'ensemble pour les techniciens, mais dans le cours de l'activité ces composantes nécessitent des choix et réclament la construction de compromis, voire d'arbitrages. Ceux-ci sont euxmêmes évolutifs, dans des situations particulières toujours à suivre en articulation avec les pratiques d'autres services, des organismes extérieurs et avec les besoins et les actions des assurés. L'incertitude est une composante forte de l'activité, car, en situation dynamique encore moins qu'ailleurs, l'organisation ne peut tout prévoir (Cellier et al., op.cit.). Il nous faut donc préciser notre approche des composantes temporelles de ces dynamiques.

\section{L'approche par les cadres temporels et leurs configurations}

Les cadres temporels, tels qu'ils ont été définis dans notre introduction, constituent tout à la fois, on l'a dit, des contraintes et des ressources potentielles dans l'activité. Ils forment un ensemble de repères qui se manifestent dans des configurations variées, et à partir desquels les travailleurs, individuellement et collectivement, structurent l'organisation de leurs actions (Grossin, 1996).

Les actions sur les cadres temporels ne consistent pas seulement à se plier à leurs exigences pour faire aboutir le travail avec la qualité attendue, mais aussi à agir sur eux pour les ajuster, ou encore les déplacer (Grossin, op.cit.). Car l'enjeu en situation dynamique est aussi d'influer sur le déroulement futur du travail, pour tenir la part d'incertitude du réel dans les frontières de ce qu'on pense - et on espère - pouvoir gérer (Pueyo, 2012).

Dans le contexte particulier des Techniciens retraite, ces cadres temporels sont tout d'abord liés aux règles et contraintes procédurales qui fixent l'échelonnement des opérations, les délais d'attente, les butées et les priorités à appliquer selon le type de demande et les caractéristiques du parcours professionnel de l'assuré. Des moyens techniques visent à soutenir ces cadres, en particulier le logiciel métier qui séquence les opérations à mener pour chaque dossier et les rappels éventuels, mais, on le verra, avec des limites. Ces cadres forts sont à mobiliser en articulation avec les demandes de l'assuré et ses réponses plus ou moins rapides aux sollicitations du technicien au fil du suivi du dossier.

Des conditions plus locales du travail liées à l'organisation dans les agences constituent une autre source de cadres temporels : les permanences d'accueil et de travail de backoffice, la quantité de nouveaux dossiers en entrée et leur mode de répartition entre techniciens, la durée des rendez-vous avec les assurés, les articulations avec le ou la référent-e d'agence et l'acheminement du courrier papier. Des cadres temporels sont liés aussi à des caractéristiques matérielles telles que les conditions d'accès à l'imprimante et aux espaces de scan partagés pour lesquels il faut se relayer.

D'autres cadres encore sont liés aux articulations avec d'autres services et en particulier le Contrôle, ces échanges étant régis par le logiciel métier, avec une procédure stricte en cas de contestation d'un retour. 
professionnels (prestation de services, mais aussi industrie, bâtiment, agriculture, etc.) ont mis en évidence l'intégration de formes temporelles de l'expérience dans l'activité, au fil du temps. L'expérience procure une appréciation élargie dans l'espace et dans le temps des évolutions des situations, ainsi que de leurs effets en termes de qualité et de performance (Weill-Fassina \& Pastré, 2004). Elle permet une appréciation des conséquences possibles de ses propres actions et de celles des autres. Ces relations sont aussi plus largement orientées dans le temps. Elles tiennent compte de ce qu'on peut apporter aux autres et des apports qu'eux-mêmes pourront constituer à plus long terme. Plus largement et pour reprendre les termes de Corinne Gaudart (2014), l'activité tend vers un enrichissement temporel et une recherche de maitrise de la perspective temporelle croissante avec l'expérience.

23 Cependant, ce développement de l'expérience ne va pas de soi, car il est « conditionnel»: il dépend des conditions de réalisation du travail au fil du temps. L'expérience résulte d'une élaboration de long cours. Elle est « le fruit d'une réflexion, 
qui fait suite au vécu d'un ensemble d'évènements, de situations dont éventuellement on peut tirer les conséquences, les enseignements " (Pueyo, 2012, p. 276) ; vivre les situations ne suffit donc pas pour constituer l'expérience. Celle-ci provient de l'action en situation, mais pour la constituer, il faut avoir pu revenir sur ces situations, y réfléchir et en tirer des enseignements, seul ou avec d'autres. Dans certaines situations de travail (changements accélérés, tâches moins stables, contraintes très serrées et enchevêtrées, etc.), où les stratégies de travail se trouvent en particulier fragilisées, dans leurs dimensions individuelles et collectives (Volkoff, 2012), l'expérience ne peut être constituée, actualisée, ou ne peut être ni mobilisée, ni transposée.

Selon cette approche, la " qualité » pour les techniciens retraite, avec ses composantes déjà mentionnées (justesse, délai, clarté, traces), dépend beaucoup des conditions de mobilisation de leur expérience dans la gestion des configurations temporelles, pour « faire avec » la complexité et l'irréductible part d'incertitude des situations évolutives qu'ils gèrent et, plus encore, pour influer sur l'évolution de ces situations.

Cette nécessité d'expertise individuelle et collective spécifique participe à l'intérêt du métier. Les techniciens que nous avons rencontrés partagent tout à la fois un fort sentiment d'utilité sociale et un intérêt pour la variété des connaissances et savoir-faire à mobiliser et pour la diversité des situations rencontrées. Il s'agit alors de mettre en évidence les conditions qui permettent à ces travailleurs de mobiliser leur expérience de la gestion temporelle en ouvrant des possibles pour l'action. Il faut aussi identifier certaines configurations qui peuvent rendre plus difficile et même entraver leur gestion de leur milieu temporel. C'était la visée principale de l'étude réalisée auprès d'eux.

\section{Une démarche de recherche et d'action}

La démarche projet s'est inscrite dans une élaboration partenariale, marquée par de nombreux échanges avec des acteurs de différents niveaux décisionnels dans l'organisme. Elle a en particulier reposé sur un comité de pilotage au rôle fort, dans le déroulement du projet comme pour la réflexion de recherche. Il a en particulier eu un rôle essentiel dans la prise en compte des enjeux, dans la mise en perspective des analyses avec les évolutions dans la branche retraite, ainsi que dans la recherche d'ouvertures pour l'action.

L'intervention s'est déroulée sur quinze mois dans quatre agences des processus Retraite, Carrières et Contrôle de différentes régions. Dans chaque agence, nous avons déroulé la même séquence d'intervention avec:

- une présentation de l'étude au responsable d'agence, puis à l'équipe avec remise d'un document explicatif de synthèse à chacun (résumé des objectifs, principe des analyses et conditions de volontariat et d'anonymat) ;

- une approche globale du travail avec visite de l'agence, et échanges informels avec les techniciens à leurs postes ;

- des analyses systématiques auprès de techniciens volontaires (modalités décrites cidessous), suivies de validations-discussions auprès d'eux ;

- et enfin des restitutions finales - discussions avec une présentation de l'ensemble de l'étude dans chaque agence, pour préciser, compléter et valider les interprétations. 
La méthodologie d'analyses systématiques a été conçue pour aborder leur activité en croisant deux modalités d'approche des dimensions temporelles :

- Des observations systématiques de l'activité de back-office ont été réalisées auprès de 12 techniciens durant une demi-journée pour chacun. Les relevés ont porté sur leurs actions, interactions (manager, collègues ou assuré), les déplacements et les outils utilisés (logiciel, téléphone, papier...), avec un repérage des dossiers examinés et de leur provenance (entrée nouvelle distribuée par le manager, courrier, courriel, appel téléphonique), et des évènements qui surviennent (appel téléphonique, retour d'un autre service en particulier dans les interactions avec le Contrôle, etc.). Les communications verbales du technicien, seul avec le chercheur ou en interaction avec d'autres, ont été enregistrées.

Chaque relevé a été transcrit sous la forme d'une chronique d'activité. Les propos et échanges verbaux ont été intégralement retranscrits, et certains extraits associés à la présentation de la chronique. L'ensemble a fait l'objet d'une analyse qualitative visant à dégager des éléments de diversité ainsi que des repères et moments structurants communs aux techniciens suivis. Ces analyses ont amené à un codage complémentaire des chroniques, pour distinguer les moments d'actions relatifs à la gestion des dossiers et aux relations aux assurés, moments qui se succèdent et parfois se chevauchent (cf. chronique 1).

31 - Des reconstitutions diachroniques du traitement de dossiers dans leurs portefeuilles ont été réalisées avec 22 techniciens, dont 2 ont aussi participé aux observations systématiques, durant des entretiens individuels $(3 / 4 \mathrm{~h}$ à $1 \mathrm{~h} 30)$. Pour chaque technicien ils ont porté sur la trajectoire de cinq dossiers, tirés un à un de leur portefeuille.

Lors de la conduite de ces entretiens, il s'agissait de recueillir les principales caractéristiques du dossier (date d'effet, phase de traitement, type de prestation), ainsi que la situation de l'assuré. L'attention a porté ensuite sur les éléments marquants dans la trajectoire et l'état actuel du dossier selon le technicien : facteurs de complexité et d'incertitude qu'il identifiait (règlementation, qualité de l'information venant de l'assuré ou spécificité de sa situation, articulations avec d'autres organismes de retraite...), appuis et difficultés du côté de l'outil technique, anticipations sur les réactions du contrôle (s'agissant des agences Carrières et Retraite), projections dans le futur et points d'attention particuliers concernant la suite possible des évènements.

Ces entretiens visaient à accéder à la pratique des techniciens à partir de cas concrets en cours dans leur portefeuille, pour identifier la mobilisation des cadres temporels jugés pertinents par eux et leurs modes d'actions sur leurs configurations. Les entretiens ont aussi constitué une fenêtre d'interruption du flux de leur activité habituelle, avec une invitation à un travail réflexif orienté vers la construction de significations et la projection dans le futur (Simpson, 2013). En suscitant une verbalisation des repères et indices utilisés pour caractériser la trajectoire du dossier et ses dynamiques saillantes, ainsi que des projections dans le futur, le questionnement du chercheur visait à identifier des configurations locales, des articulations dynamiques, et des modes d'action individuelle ou collective sur le milieu temporel, avec des mises en perspective faites de particularisations ou de généralisations.

- Ces deux méthodes se sont ensuite rejointes dans une phase de validationsdiscussions d'auto-confrontation individuelle, avec chacune des personnes 
interrogées ou suivies. Cette phase s'est appuyée sur les premières analyses des relevés réalisés auprès de chacun (chroniques d'activité ou entretiens sur portefeuille), en proposant au technicien de les commenter en tout ou partie pour ajuster et approfondir les interprétations.

$\mathrm{Au}$ total, 76 personnes travaillant en agences ont été concernées par nos analyses, dont 6 managers d'agence, et 32 techniciens directement impliqués dans les analyses systématiques et les restitutions. Les analyses réalisées auprès des techniciens dans les agences ont été alimentées par des retours - discussions réalisées dans plusieurs instances qui ont jalonné le projet: des ateliers de managers et de directeurs métier, le Comité de direction des RH de la branche, une direction régionale, un CHSCT régional, ainsi que le Comité de direction de l'organisme. Les échanges dans ces instances prenaient appui sur les analyses en cours, tout en les alimentant en retour et en traçant des ouvertures vers des pistes d'action.

Après remise du rapport final, les contacts se sont poursuivis, en particulier pour des échanges concernant la mise en place d'actions. Elles ont été mises en débat avec des acteurs de différents niveaux décisionnels. Les résultats - dont nous allons maintenant présenter des extraits - ont aussi été alimentés par des restitutions-discussions auprès de plusieurs groupes de responsables et de représentants des salariés.

\section{Comment les techniciens conseil gèrent leurs cadres temporels}

37 L'ensemble des situations analysées a mis en évidence de nombreuses formes de l'activité déployée par les techniciens. Qu'ils soient du métier Retraite, Carrières, ou Contrôle, ces formes se traduisent par un foisonnement d'actions et d'interactions qui se succèdent ou s'entremêlent, en reflétant la prise en compte et l'organisation de cadres temporels nombreux et variés, dans l'activité. Nous commençons ici par montrer comment ces actions se gèrent au fil des configurations temporelles d'une journée. Nous prendrons ensuite trois configurations plus transversales de leur activité afin d'analyser, pour chacune d'elles, les modes de gestion des cadres temporels et les enjeux que cette gestion recouvre en termes de qualité.

\subsection{Les configurations temporelles des journées de travail : organiser l'ouverture des dossiers}

38 Les douze plages d'observation sont de durées variables et ne font pas ici l'objet de comparaisons. Le nombre de dossiers ouverts par les techniciens l'était également (4 à 21), ainsi que la durée des traitements réalisés sur chacun (de 1 à $50 \mathrm{~min}$ ). Parmi eux, 13 ont fait l'objet d'un échange Contrôleur -technicien Retraite ou Carrière suite à une invalidation du contrôleur (Tableau 1 : colonne « dont retours en cours »). 
Tableau 1 : Dossiers traités par les techniciens Retraite, Carrières et Contrôle durant les 12 périodes d'observation (hors scan pour numérisation).

Table 1: Case files processed by Retirement, Career and Control technicians during the 12 observation periods (excluding scanning for digitization)

\begin{tabular}{|c|c|c|c|c|c|}
\hline & Observations & $\begin{array}{l}\text { Dossiers ouverts } \\
\text { et examinés }\end{array}$ & \begin{tabular}{|c|} 
dont retours \\
en cours
\end{tabular} & $\begin{array}{l}\text { Fourchette de } \\
\text { durées }\end{array}$ & $\begin{array}{l}\text { Dossiers validés avec } \\
\text { clôture de phase portefeuille }\end{array}$ \\
\hline \multirow{8}{*}{$\begin{array}{l}\frac{D}{\pi} \\
\frac{\pi}{2} \\
\frac{\pi}{0}\end{array}$} & R-J1matin ${ }^{(1)}$ & 11 & 0 & 3 à $45 \mathrm{mn}$ & $\begin{array}{l}2 \text { acceptés par le contrôle automatique } \\
\text { (analyse des risques financiers) }\end{array}$ \\
\hline & R-J1aprèm & 9 & 1 & 8 à $20 \mathrm{mn}$ & 7 envoyés au Contrôle \\
\hline & R-J2matin & 21 & 3 & 1 à $27 \mathrm{mn}$ & $\begin{array}{l}3 \text { acceptés par le contrôle automatique } \\
2 \text { envoyés au Contrôle }\end{array}$ \\
\hline & R-J2aprèm & 11 & 1 & 1 à $20 \mathrm{mn}$ & 8 envoyés au Contrôle \\
\hline & R-J3matin & 11 & 0 & 5 à $23 \mathrm{mn}$ & 0 \\
\hline & R-J3aprèm & 11 & 2 & 2 à $32 \mathrm{mn}$ & 1 accepté par le contrôle automatique \\
\hline & RJ4matin (2) & 13 & 3 & 3 à $30 \mathrm{mn}$ & 1 envoyé au Contrôle \\
\hline & R-J4aprèm & 8 & 0 & 4 à $35 \mathrm{mn}$ & 1 envoyé au Contrôle \\
\hline \multirow{2}{*}{ U. } & Car-matin & 5 & 1 & 7 à $24 \mathrm{mn}$ & 1 envoyé au Contrôle \\
\hline & Car-aprèm & 4 & 0 & 5 à $30 \mathrm{mn}$ & 1 accepté par le contrôle automatique \\
\hline \multirow{2}{*}{$\begin{array}{l}\frac{0}{0} \\
\frac{2}{2} \\
0 \\
0 \\
0\end{array}$} & Co-matin & 8 & 2 & 4 à $33 \mathrm{mn}$ & $\begin{array}{l}3 \text { validations } \\
4 \text { invalidations -retours }\end{array}$ \\
\hline & Co-aprèm & 4 & 0 & 30 à $50 \mathrm{mn}$ & $\begin{array}{l}3 \text { validations } \\
1 \text { invalidation -retours }\end{array}$ \\
\hline
\end{tabular}

Notes 1 et 2 : voir les chroniques d'activité de même numéro.

Note 3 : Dans cette colonne on comptabilise :

- pour les techniciens Retraite ou Carrière, les dossiers invalidés, en retour du service contrôle ; - pour les Contrôleurs, les dossiers qu'ils ont invalidés sur lesquels ils échangent avec des techniciens Retraite ou Carrière.

L'ensemble des demi-journées de suivi a permis de couvrir des situations d'actions diverses correspondant à différentes facettes du travail. Ces situations ont permis de souligner la multiplicité des actions qu'ils réalisent et une organisation en différents moments qui correspondent d'une part aux modalités par lesquelles les dossiers ou des documents complémentaires leur parviennent, et d'autre part aux interactions avec les assurés, qu'ils entrelacent avec le traitement de ces dossiers (cf. exemple en Chronique d'activité 1: Actions «Traitement des dossiers " et "Gestion de l'assuré»). Ces moments d'action sont engagés et se succèdent selon une organisation d'ensemble propre à chaque technicien. Chacun explicite spontanément cette organisation, comme en début de matinée dans la Chronique : «La première chose que je fais est voir [le courriel], si je n'ai pas de messages importants, sur les dossiers urgents [...]». Dans cet exemple, la technicienne a pris connaissance des courriels, puis réalisé des opérations sur six dossiers en entrée (Dossiers 1 à 6), classé par priorité les dix suivants et entamé leur traitement (Dossier 7 à 11).

$\mathrm{Au}$ total, 11 dossiers sont traités ce matin-là, dont 2 seulement peuvent être validés en vue de leur liquidation tout en devant encore être suivis, car il faut attendre le retour du courrier adressé à l'assuré, prendre en compte les dernières informations, puis s'assurer de la validation du service Contrôle. Ainsi, ces 11 dossiers restent à suivre pour la technicienne, ce qui est une situation courante. Lors de nos observations, jusqu'à 21 dossiers ont été ouverts par demi-journée dont une petite proportion a été 
envoyée au Contrôle (cf. Tableau 1), tout en restant souvent à suivre quelques jours dans le portefeuille. Dans tous les cas, ce suivi complémentaire n'étant pas soutenu par le logiciel, il nécessite de la part de chaque technicien, outre la mise en attente du courrier papier, un mémo associé (souvent papier) rappelant les numéros d'assurés encore à suivre.

Figure 1 : Chronique d'activité 1 - Exemple d'une matinée d'un technicien retraite : une activité dynamique de gestion des dossiers articulée avec le suivi des assurés (Zara-Meylan \& Volkoff, 2019) - Relevés traités avec ActoGraph ${ }^{\circledR}$.

Figure 1: Activity chronicle 1 - Example of a pension technician's work morning: the dynamic management of case files activity, combined with beneficiary follow-up (Zara-Meylan \& Volkoff, 2019) records processed using ActoGraph ${ }^{\circledR}$

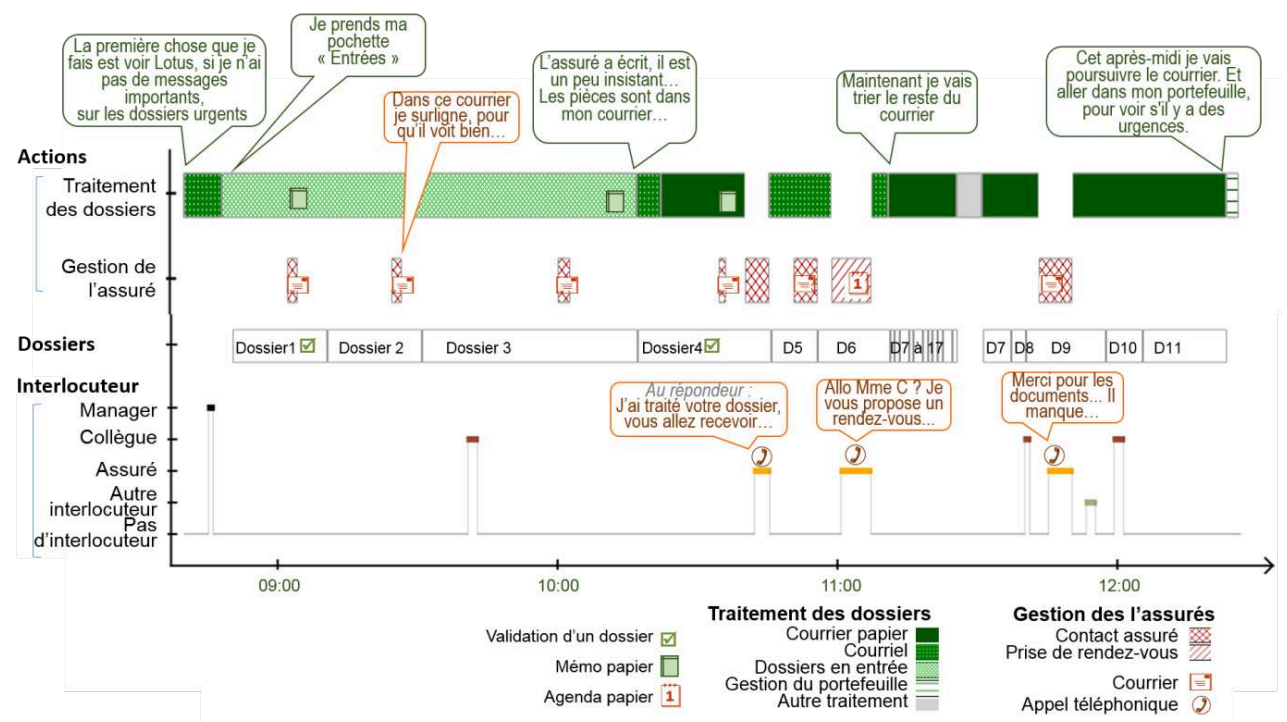

Durant cette matinée, de nombreux dossiers amènent la technicienne à des contacts avec un assuré. Certains nécessitent un courrier d'information ou une demande de pièce complémentaire (dossiers 1 à 5 , puis 9), dans laquelle la technicienne prend soin de surligner des mentions importantes, en rayant celles qui pourraient prêter à confusion. D'autres dossiers (ou les mêmes) amènent à joindre l'assuré au téléphone, comme le dossier 4 qui concerne un assuré insistant et inquiet, le dossier 5 pour fixer un rendez-vous, et le dossier 9 pour lequel la technicienne appuie par un appel téléphonique sa demande écrite de pièce complémentaire.

L'ensemble des observations montre que les techniciens organisent leur travail et s'organisent dans des modalités qui incluent, tout en les dépassant largement, les fonctions proposées par le logiciel métier ${ }^{2}$. Ils organisent leur travail au fil des journées et pour chaque dossier en prévoyant l'échelonnement des actions à mener, dont la consultation de la fonction "pilotage du portefeuille » et des alertes du logiciel ; et ils s'organisent et se réorganisent pour garder ouvertes des possibilités d'agir dans la diversité des évènements et aléas qui peuvent survenir.

Pour cela ils mobilisent des configurations d'organisation constituées d'une palette de repères, d'échelles et d'horizons complémentaires. "C'est de la gestion au quotidien, et de la gestion hebdomadaire (...), mais un peu plus aussi je dirais, parce que j'ai préparé mes rendezvous à 3 semaines ", explique l'un d'eux (TCR) ${ }^{3}$. Un autre évoque un horizon glissant sur un mois : "à 30 jours, on peut penser que ce dossier sera hors délai, penser à mettre un motif d'attente... » (TCR). Ces repères permettent à la fois une régularité d'actions et 
d'interactions, et des ajustements pour intégrer les évènements qui peuvent survenir, essayer d'en déplacer d'autres, décider d'une relance, appeler un assuré afin de susciter une action de sa part, ou boucler un dossier avant d'obtenir une pièce. Ils sont structurants pour l'activité individuelle et collective des techniciens, tout en étant structurés par celle-ci.

\subsection{Les configurations temporelles de construction du relevé de carrière : consolider au fur et à mesure}

Le traitement du dossier d'un assuré comporte plusieurs étapes. La première, qui peut être renouvelée à plusieurs dates, est la reconstitution de carrière ou " régularisation ". Elle prend place en amont de la phase de liquidation, ou se mène conjointement avec celle-ci quand l'assuré dépose sa demande d'ouverture des droits, ce qui est fréquent. En termes de cadres temporels, ces deux cas de figure sont très différents et concernent des métiers distincts.

Pour les techniciens carrière, il s'agit en général de «campagnes » de régularisations, impulsées par la direction des données sociales, avec des cibles générationnelles, ou par secteur, par métier, par type de parcours. Les techniciens vérifient et complètent les dossiers des assurés qui répondent à ces campagnes et qui sont, a priori, encore loin de leur date de liquidation.

Il n'en va pas de même quand la régularisation intervient au moment même de la demande de liquidation. Cette fois, ce sont les techniciens retraite qui sont en charge du dossier, et se montrent évidemment attentifs à la date d'effet de la demande, qui conditionne les délais disponibles pour régulariser le relevé.

Dans les deux cas, le but poursuivi est d'établir des relevés impeccables, sans béances, avec des documents attestant de la véracité des renseignements pour chaque période. La raison principale est que ce relevé va fonder les calculs de droits à pension, pour le reste de l'existence des intéressés; une raison complémentaire est que ce relevé peut être consulté par la suite, en cas de contestations ou lors de calculs de pensions de réversion par exemple.

Cette reconstitution des carrières ne relève pas de l'application mécanique de procédures. Elle nécessite des explorations parfois complexes : démarches auprès des archives, employeurs qui n'existent plus, organismes difficiles à joindre, ou à l'étranger, etc. Dans bien des cas, la notion même de montant « erroné » ou de parcours "à vérifier» implique une marge d'appréciation, qui comporte des dimensions temporelles. En témoigne cet échange :

... si je me mets sur 2012, je considère que le salaire est bon, je fais un clic droit, " certifié" .

Qu'est-ce qui pourrait faire que le salaire ne soit pas « bon »? (ergonome)

Là, voyez, c'est quelqu'un qui est en progression constante. Si je me mets sur les... disons, les six dernières années...le $n^{\circ}$ employeur est le même, ça nous paraît logique comme évolution

de salaire... il n'y a pas de souci de no employeur, de choses comme ça, donc c'est bon. (TCR)

La technicienne a examiné un empan de six ans, mais elle ajoute : «... disons ». C'est un choix de sa part, élaboré en prenant en compte des règles de métier, des particularités de l'itinéraire de cet assuré, l'allure générale de son parcours, et les évènements qui l'émaillent. Le critère de la "progression constante » a joué un rôle rassurant, mais il est fréquent qu'il ne soit pas rempli ; en outre une progression qui se poursuit, mais change soudain de rythme peut, elle aussi, susciter des doutes. Entre les cas 
manifestement bizarres et ceux qui sans encombre sont considérés normaux, une zone intermédiaire existe, où s'exerce le jugement du technicien, assorti d'un pari sur la vision qu'en aura le technicien contrôle, en aval.

Or ces explorations et raisonnements s'effectuent au gré des actions multiples décrites ci-avant (cf. 4-1), sous une pression temporelle qui tend à s'accentuer, et face à des assurés dont les carrières sont de plus en plus compliquées, ce qui les incite souvent selon les techniciens - à poser leur demande tardivement après de longues hésitations. Ce dernier aspect peut mettre en difficulté les techniciens retraite, qui ont à l'esprit l'impératif de continuité des ressources pour les assurés. La pression s'exerce aussi sur les techniciens carrière, dont on aurait pu croire qu'ils en seraient davantage abrités :

... je l'ai clôturé en mettant "dans l'attente du contrat d'apprentissage». Et quand je recevrai ce contrat je rouvrirai le dossier.

Quel est l'intérêt de clôturer? De ne pas laisser trop traîner... (ergonome)

Parce que, eh bien on est... (rires)... on est tenu à garder les dossiers deux mois au plus (vers une collègue : c'est ça ?) (TCar)

51 Lorsque les consignes relatives aux délais de maintien en portefeuille, ou aux quantités à traiter par semaine, se renforcent, il devient difficile de dégager des temps pour vérifier et approfondir le traitement de certains dossiers. Plusieurs techniciens Carrières ont exprimé la crainte que la qualité des dossiers en pâtisse, qu'il faille par la suite revenir sur les régularisations bouclées trop rapidement, et que ces rattrapages ne soient pas toujours réalisables - eu égard aux contraintes de temps, déjà décrites, des techniciens retraite :

[...] Bon, on nous dit toujours qu'au moment du départ en retraite le technicien retraite revoit tout ça. Je pense que le technicien retraite, il est comme nous, il doit avoir énormément de dossiers à traiter [...]. Quand on dit que la retraite est payée au juste droit, moi je décline !! Je pense qu'il y a des reports manquants. (TCar)

Pour gérer, dans ce contexte tendu, les cadres temporels de la reconstitution de carrière, les techniciens peuvent compter sur deux points d'appui principalement : les collègues en amont, et les assurés eux-mêmes.

Les opérations qu'ont effectuées des collègues quelques mois ou quelques années auparavant, surtout si elles ont été contrôlées ${ }^{4}$, soulagent énormément le technicien qui reprend le dossier, et même le contrôleur qui interviendra à sa suite :

... des fois on va voir que cela a été remonté par régularisation, après on peut

constater que ça été validé par un contrôleur, alors là on ne s'en occupe pas (TCo).

Comme le resserrement des contraintes sur les techniciens carrière les amène de plus en plus souvent à laisser en suspens une partie de leurs ajouts ou de leurs vérifications, c'est de la coopération avec l'assuré que va largement dépendre la gestion des temps dans la phase de liquidation. Le plus souvent cette coopération tire parti d'un climat de confiance réciproque, et d'une attitude très proactive du technicien, qui reprend à son compte les souhaits et espérances des futurs retraités, et se félicite des avancées. En ont témoigné pour nous les propos d'une technicienne qui vivait comme un "coup de bol " une validation de trimestres, "juste au nombre que je recherchais»; ou ceux d'une autre qui, parlant d'une assurée à carrière incomplète et petit salaire essayant de tirer le meilleur parti de sa dernière année d'activité, a utilisé tour à tour les expressions : « elle court après les trimestres ", " je cours après les trimestres » et " on court après les trimestres ". Toutefois cette coopération n'est pas toujours harmonieuse et efficiente. L'assuré est plus ou moins disponible, plus ou moins réactif (surtout à l'égard des techniciens 
carrière, quand lui-même est loin de sa retraite), plus ou moins avisé de ses droits, plus ou moins clair dans ses explications. Certains engagent leurs démarches un peu tard et, comme nous l'avons signalé, ce sont souvent ceux dont la carrière est compliquée. D'autres, dans le doute, adressent à l'agence une pléthore de documents superflus, une pratique que les techniciens connaissent bien et baptisent «l'envoi de boîtes à chaussures".

Dans ces conditions, la gestion du milieu temporel repose sur des stratégies subtiles pour amener un assuré à accomplir sans tarder les tâches que l'on attend de lui. Cela consiste par exemple à ne pas lui annoncer par avance l'échec de sa demande de liquidation à la date souhaitée, faute de droits suffisants. Le but est de ne pas le démobiliser d'emblée, afin d'obtenir tout de même de lui les documents nécessaires à la mise à jour de sa carrière :

C'est possible que cette assurée ait droit à la retraite carrière longue ? (...) Sinon, vous lui diriez, d'emblée ? (ergonome)

Non. Je le fais à la fin. Je ne vais pas lui dire qu'elle n'y aura pas droit, parce qu'elle ne me renverra jamais ses documents.

Vous voulez les avoir, ces documents?

Eh bien, comme ça (...), quand elle déposera son dossier, sa carrière sera quasiment à jour. Le but c'est que les dossiers de retraite, ça coule de source. (TCR)

$57 \mathrm{Au}$ cœur de la gestion du milieu temporel dans les phases de régularisation de carrières, on retrouve donc en permanence des arbitrages entre un souci de précision et le respect de butées temporelles plus ou moins explicites : dans quels cas juger qu'un relevé est normal, ou au contraire mérite des vérifications? Quand boucle-t-on un dossier, ou au contraire choisit-on de (re)demander des renseignements? Parmi ces renseignements, lesquels sont indispensables? Cette demande effectuée, pendant combien de temps attendre la réponse ? Il y a là des facteurs d'incertitude, qu'une technicienne commente ainsi :

(...) j'ai été longtemps à relancer, là, sur des machins, on me l'a assez reproché, qu'on ne clôture pas assez vite, qu'on s'attarde trop sur des choses (...) comme on nous dit qu'il faut qu'on arrête de retourner des trucs sans arrêt... donc moi j'ai commencé à prendre l'habitude, à peser le pour et le contre. (TCar)

Des règles orientent en partie ces arbitrages, mais elles ne peuvent prévoir la très grande diversité des situations personnelles (les «choses " qu'évoque la TCR), ni celle des configurations temporelles dans le traitement des dossiers ; et leur usage évolue, ce qui refaçonne les stratégies adoptées par chacun («donc moi j'ai commencé à...»), avec cependant peu d'espaces pour échanger entre collègues sur la gestion adoptée et ce qu'elle met, même provisoirement, de côté.

\subsection{Les configurations temporelles d'installation d'un rendez-vous : accorder du temps au bon moment}

Nos observations ont porté presque uniquement sur l'activité de back-office, mais nous avons recueilli de nombreux éléments sur la préparation des rendez-vous avec les assurés, et sur le rôle clé que ces rendez-vous peuvent jouer. Le choix de provoquer un rendez-vous, de l'accepter ou le différer, la date choisie, son ordre du jour, la conduite même de l'entretien, constituent des composantes cruciales de la gestion du milieu temporel dans leur activité. 

cette occasion, la décision de rendez-vous se prend avec prudence. Le trajet, suite notamment aux fusions d'agences, peut être long pour l'assuré. Certains ont besoin d'être accompagnés de personnes de leur entourage. Il faut donc que le déplacement en vaille la peine. En outre le rendez-vous peut créer chez l'assuré l'idée qu'ensuite la procédure va s'accélérer, voire que l'aboutissement est proche. Cette idée est en partie fondée : "après le rendez-vous, tout se met en route » (TCR). Mais ce n'est pas certain. D'où l'intérêt de ne pas placer cette entrevue trop tôt si l'on pressent des obstacles :

«il y avait la MSA', de toute façon, qui me bloquait. Je ne voyais pas l'intérêt de faire déplacer l'assurée pour venir me porter un document, sachant que je ne pourrais pas aller plus vite » (TCR).

61 Une fois décidé, le rendez-vous est préparé de part et d'autre. Comme on l'a signalé au paragraphe précédent, il est usuellement fixé trois semaines à l'avance environ, avec au besoin des échanges téléphoniques préalables, notamment pour obtenir de l'assuré qu'il rassemble les documents nécessaires. Le technicien, de son côté, établit souvent une fiche récapitulative informelle, "comme un petit pense-bête»; il la relit avant la rencontre et l'apporte avec lui dans le box de rendez-vous. de latence: par avance, puisque la date de rendez-vous constitue une butée pour l'assuré qui doit s'y préparer; et après coup, parce que les incompréhensions techniques et les hésitations chez l'assuré sont plus faciles à gérer en face à face, ce qui amoindrit les difficultés par la suite.

63 C'est pourquoi les techniciens insistent sur la fonction de gestion temporelle que les rendez-vous peuvent remplir. Dans cette agence, où les entretiens venaient d'être fixés à 30', au lieu de 40' auparavant, une technicienne explique le rôle du rendez-vous en tant que moment-clé d'un dossier bien monté, aussi bien pour son contenu que pour la maitrise des délais ensuite :

Les dossiers des gens qu'on reçoit en rendez-vous sortent plus vite, parce qu'on a pris le temps de discuter, même si on n'a pas pu aller vraiment en profondeur (...) C'est aussi parce que, quand les gens viennent, on leur dit de venir avec tel document, donc les dossiers sont quand même relativement complets. (TCR)

Le rendez-vous n'est pas seulement le lieu où se scellent les informations et les décisions pour le départ en retraite ; c'est aussi l'espace d'un arbitrage encore possible :

La personne dont je vous disais qu'elle est restée une heure, je croyais que c'était un dépôt de dossier, je lui avais envoyé le document habituel. Et quand elle est arrivée, elle me dit «je ne veux pas déposer mon dossier tout de suite, je veux avoir le maximum de surcote... », c'est vraiment devenu un entretien-conseil. (TCR)

Ce double objectif, de fixer solidement les éléments du dossier et d'offrir des marges de manœuvre pour son adaptation, constitue aussi un double argument, selon cette technicienne, pour ne pas normer de façon trop stricte la durée des rendez-vous. Ce qui l'amène à passer en revue de nombreux facteurs de variabilité :

Parfois (...), la personne n'aura pas énormément de questions à poser, (...) elle aura fait sa reconstitution de carrière, ce sera juste une formalité : le dépôt de la demande, prendre ses papiers, enregistrer, lui donner son accusé de réception, ça va prendre $10^{\prime}$ ou $1 / 4$ d'heure. Alors que d'autres dossiers, (...) ce sont des gens en difficulté, parfois ils ont du mal à comprendre les démarches administratives, les papiers (...). Il y en a aussi qui sont pointilleux, ils veulent savoir en détail comment se fait le calcul, pourquoi telle année n'est pas prise dans le calcul du SAM ${ }^{6}$. (...). Si c'est une pension de réversion, des gens qui viennent de perdre leur conjoint, il faut 
prendre le temps, ne pas les brusquer. Quelquefois aussi, il y a un rendez-vous qui déborde, quand par exemple un couple vient, Monsieur dépose son dossier de retraite, et la dame me dit « ah bien moi justement, j'ai une question à vous poser sur mon relevé de carrière... ». (TCR)

Selon cette technicienne la réduction de 10 minutes ne raccourcissait pas en réalité le temps nécessaire à un rendez-vous, et ne répondait donc pas aux objectifs gestionnaires invoqués par l'organisme :

Les rendez-vous qui prenaient moins de temps nous permettaient de finaliser le dossier juste après, et quand on revenait au bureau on n'avait plus qu'à scanner la demande. (TCR)

Les fenêtres qui permettaient de terminer certains traitements au cours des rendezvous ont disparu. Et tous les dossiers nécessitent désormais une finalisation de retour au bureau.

Ajoutons ici qu'un rendez-vous bien mené, sans encadrement temporel trop étroit, donne la possibilité de développer la compréhension de l'assuré sur les règles et procédures du système de retraite dans son ensemble, ce dont peuvent à leur tour bénéficier des membres de son entourage. Le gain d'efficacité que cela peut représenter pour la branche, en termes de qualité du travail effectué par les assurés eux-mêmes, mériterait d'être apprécié - ce que les indicateurs usuels de performance ne peuvent repérer.

\subsection{Les configurations temporelles d'interactions avec le contrôle : incorporer les signalements}

69 Une dimension de l'activité concerne les relations avec le Contrôle, en particulier lors d'un retour effectué par un Contrôleur au technicien Retraite ou Carrière. Treize situations de ce type ont été observées. Pour comprendre comment le traitement de ces retours s'inscrit dans l'activité, examinons un extrait d'une de ces situations.

En faisant état de son mode d'organisation, cette technicienne indique tout d'abord la place importante de la consultation des retours du contrôle dans son activité :

« Là, tous les matins je regarde le portefeuille. C'est la première chose que je fais, les retours... Bon il n'y en a pas toujours (rires)».

Puis elle examine les trois retours qui viennent de lui arriver du Contrôle :

$-. . . / .$.

- 8 h 54 : Dossier 1. Le TCR lit, examine le dossier, parcourt différentes pages

écran.

«Donc là, je ne suis pas forcément d'accord avec le retour.»

Cherche dans la documentation en ligne : «J'essaye de trouver... Bon, alors... » Calcule à mi-voix avec calculette. «Eh bien je ne suis pas d'accord. Bon, je pense qu'il faudra que j'aille voir ma conseillère technique.»

- 9 h 2 : Dossier 2. Le TCR lit, examine le dossier, parcourt différentes pages écran. "Je ne vais pas être d'accord non plus. C'est un fonctionnaire. Donc il fallait remonter les trimestres fonction publique, ce que j'ai fait [pour une question de délai]. Sauf qu'entretemps ils l'ont fait aussi de leur côté ».

- 9 h 6: Dossier 3. Le TCR lit, examine le dossier. «Là c'est une ressource, ils [le contrôle] me disent que "le montant est erroné" "

"Je ne comprends pas non plus parce que pour moi c'est bon. [...] j'ai le justificatif. J'ai remonté ce que j'avais. "

- 9 h 8 : Place ces trois dossiers papier dans une bannette: « Donc là, je pense que ces 3 retours ne sont pas justifiés. Il faut que je voie avec [la référente technique]. » 
$-\ldots / \ldots$

71 Le TCR met donc les 3 dossiers de côté, car elle doit en parler avec la référente technique de l'agence, qui pourra elle-même se mettre en relation avec le Contrôleur. Le manager sera aussi sollicité pour des régulations avec le service Contrôle sur l'un de ces retours.

72 Les dossiers 1 et 3 s'avèrent liés à des incompréhensions entre le TCR et le Contrôleur. Les TCO apportent du soin à leurs retours, mais les interfaces ne sont pas les mêmes pour les deux métiers, le dossier et ses points saillants ne se présentent pas de la même façon, et la procédure impose des échanges entre TCR et TCo uniquement via l'outil logiciel. Les coups de fil étant supposés se traduire par des perturbations pour le travail des contrôleurs, ils font partie des pratiques du passé désormais prohibées, sauf s'ils sont à l'initiative de ces derniers. Nous avons pu constater pourtant que ces échanges verbaux permettent de lever rapidement les doutes et de résoudre les incompréhensions, mais ils restent l'exception.

73 Au sujet du dossier 2, la technicienne explique qu'elle a complété manuellement des trimestres effectués chez un employeur public afin de ne pas risquer un " hors délai ». En effet, un dépassement de délai (fixé par les indicateurs de gestion) pénaliserait le technicien, mais aussi l'assuré dont la pension serait différée. Dans ce cas, il se trouve que l'employeur a finalement aussi complété le dossier juste après, ce qui a amené le Contrôle à signaler une "erreur pour double report». Comme dans de nombreuses situations, le technicien a pris une décision en estimant les évolutions futures de la situation. Ces décisions participent le plus souvent aux dimensions de la qualité qu'ils produisent, mais dans certains cas, comme celui-ci, le dossier évolue encore dans les quelques jours qui séparent sa validation et les vérifications du Contrôle, ce qui déclenche un retour en « erreur ».

74 De plus, de nombreuses prescriptions transitoires, relayées par des notes de service, sont relatives à des évolutions règlementaires non encore prises en charge par le logiciel. Certains types de dossiers sont donc à traiter manuellement, selon des consignes et cadres temporels spécifiques (par exemple: considérer les nouveaux critères de carrières longues, suspendre quelques jours des dossiers d'assurés aux années de naissance charnière lors de l'allongement de la durée des cotisations, ou effectuer des interrogations pour certains parcours à l'étranger). Ces procédures transitoires constituent une préoccupation supplémentaire pour les techniciens qui doivent identifier ces dossiers non signalés par le logiciel et penser à se référer aux consignes qui s'empilent, au risque d'un retour en erreur en cas d'oubli. Elles contribuent à multiplier les cadres temporels à gérer.

Les analyses mettent ainsi en évidence différentes combinaisons de conditions techniques et organisationnelles qui compliquent ou fragilisent la gestion de multiples dimensions temporelles assurée par les techniciens. Les retours du Contrôle traduisent en particulier certaines de ces difficultés. En pareil cas le terme « d'erreur » par lequel ils sont qualifiés constitue une appellation discutable, décalée avec la réalité des enjeux de qualité pourtant repérés et tenus par le technicien, à la fois pour le dossier et pour l'assuré. 


\section{Quelques pistes de réflexion}

tout au long de l'étude, un positionnement vis-à-vis de la notion de qualité. Ce positionnement a été explicité auprès des demandeurs au niveau de la branche retraite comme en agence, en proposant au-delà des indicateurs de qualité usuels un sens élargi de la qualité. Pour cela, les dimensions de justesse de la prestation, de respect des délais, de clarté pour l'assuré et de qualité des traces laissées dans les dossiers, que nous avons identifiées (cf. 1.1) ont constitué de bons supports, en amenant des échanges ouverts sur l'activité. De plus, en montrant comment ces facettes de la qualité se trouvent étroitement liées aux possibilités des techniciens de suivre et d'organiser les multiples dynamiques de leurs situations en tenant compte de cadres temporels variés et évolutifs, nous établissions au passage qu'une grande part de cette contribution est difficilement accessible à la quantification. Et nous suggérions au fur et à mesure de nos échanges à différents niveaux (managers, ateliers de travail, comités de pilotage, comité de direction national) d'adopter une acception élargie de la qualité du travail, et des modes d'appréciation de cette qualité qui soient distincts des indicateurs de gestion.

versés aux échanges ont permis de préciser la nature de l'activité des techniciens qui, loin de se résumer en une juxtaposition d'actions cloisonnées sur différents dossiers, doit être comprise dans la gestion construite qu'ils assurent, individuellement et collectivement. Cette gestion temporelle multimodale et son étendue sont pourtant méconnues des prescriptions comme du logiciel métier, qui classiquement préconisent une succession de phases du travail, considérant l'activité dans une temporalité rétrécie (Gaudart, 2014). Les actions des techniciens, certes organisées à l'échelle des journées, visent tout autant une cohérence sur le long cours et des ouvertures de possibles pour leurs actions. Par ces appréciations, ces actions et perspectives d'actions, les techniciens appréhendent et façonnent les multiples configurations composées des cadres temporels, mais aussi de leurs interrelations évolutives (Elias, 1993). Avec l'expérience ils appréhendent ces configurations dans une 
actualité associant passés (y compris les voies non empruntées), présents et futurs envisagés. Cela leur permet de tenir compte des évolutions en cours des configurations, des effets de leurs actions (y compris des attentes) et des évolutions futures comme des possibilités d'actions qu'ils envisagent (Zara-Meylan, 2016).

Dans ce contexte, la qualité du pilotage des dossiers au fil de leur itinéraire, dans un service ou d'un service à un autre, doit beaucoup aux relations que les techniciens parviennent à instaurer avec les assurés en tenant un ensemble de contraintes liées à l'organisation et en positionnant une relation sociale avec chacun, et plus encore en les enrôlant dans la gestion des cadres temporels, comme l'étaient par exemple les patientes âgées dans la recherche d'Esther Cloutier auprès des aides à domicile (Cloutier, David, Teiger, \& Prévost, 1999). Les relations de services sont souvent abordées en privilégiant leurs dimensions expressives et affectives : l'injonction faite aux agents de "trouver la bonne distance ", entre indifférence et empathie, entre souci d'apaisement et défense de sa dignité dans les situations de conflit (Jeantet, 2003 ; Molinier, 2009). Or notre analyse de leur activité suggère que d'autres «distances » (Volkoff, Zara-Meylan, Mardon, \& Gaudart, 2016), spatiales, cognitives et surtout temporelles sont tout aussi importantes à régler et que ce réglage en continu constitue une composante majeure des dynamiques de la relation de service.

81 Une autre ressource majeure pour la gestion temporelle est le développement de pratiques réflexives avec les collègues, l'encadrement, les référents techniques, à propos de situations d'hésitation ou d'incertitude. Ces échanges sont constitutifs de l'expérience et dotent les travailleurs de ressources renforcées pour effectuer des choix qui orientent le déroulement de configurations temporelles pertinentes, prenant appui sur leurs expériences passées, et sur des savoirs professionnels dont ils peuvent faire profiter leurs collègues actuels. Une première conclusion générale de cette recherche est donc que la gestion pertinente des cadres temporels dans un objectif de qualité dépend des possibilités de faire connaitre et reconnaitre les besoins et enjeux de cette activité, ainsi que des conditions dans lesquelles des échanges de savoirs et de pratiques peuvent ou non s'établir entre travailleurs d'un service et, au-delà, entre différents métiers.

82 Par ailleurs, les analyses amènent à insister sur la question de l'ajustement entre les règles qui soutiennent un enjeu de rigueur administrative et d'homogénéisation des procédures pour l'organisme, et la diversité des configurations, en particulier temporelles, à gérer. Un effort d'appréciation des processus en cours toujours spécifique en découle pour les techniciens avec: des suppositions quant au comportement passé et à venir de tel interlocuteur, en particulier l'assuré lui-même ; des raisonnements, par exemple pour évaluer l'urgence d'une relance à faire, en tenant compte d'articulations souvent incertaines avec des services ou organismes extérieurs.

Ces ajustements nécessitent parfois un arbitrage entre des prescriptions sources de cadres temporels non conciliables dans l'immédiat. On l'a vu, il s'agit par exemple de viser tout à la fois la complétude d'un relevé et la nécessité de ne pas y consacrer trop de temps, d'attendre la confirmation d'un employeur, mais respecter des délais, ou encore demander une pièce complémentaire et gérer un portefeuille devenu trop lourd $\mathrm{du}$ fait du grand nombre de dossiers en attente. Comme dans des centres de services partagés où des gestionnaires sont supposés établir des payes de qualité et « rendre le client satisfait » sans pouvoir contacter les donneurs d'ordre, et en étant surtout évalués sur la quantité traitée (Arnoud \& Falzon, 2013 ; Arnoud, Krohmer, \& Falzon, 
2018), ce sont des situations porteuses de dilemmes, qu'on peut ici qualifier de temporels.

Les conditions de résolution de ces dilemmes ne sont parfois pas facilitées par les prescriptions et modes d'organisation. Dans les centres de services partagés, les tâches sont de plus en plus parcellisées et les gestionnaires sont supposés pister et consigner les manquements des clients par rapport au contrat de partenariat, alors que les analyses montrent l'importance de la coopération en interne et avec le client pour comprendre, se mettre d'accord et pour assurer une qualité d'ensemble (Arnoud et al., 2018). De même, dans notre recherche, les modes d'organisation qui cloisonnent géographiquement et techniquement le traitement des dossiers et le contrôle ne facilitent pas la résolution des dilemmes dans l'organisme retraite. Le mode de retoursanction du Contrôle vers les techniciens Carrière ou Retraite réduit et entrave des échanges, alors que ceux-ci, nous l'avons montré, s'avèrent souvent fructueuses en participant à alimenter l'expérience de part et d'autre. Quand l'organisation méconnait l'apport des débats et des échanges à propos des dilemmes temporels, elle peut placer les travailleurs dans des situations paradoxales (op.cit.) où, ne pouvant échanger au sujet des dilemmes, ils sont amenés à encourir des risques pour eux-mêmes (nonrespect des procédures, des délais, moindre quantité de dossiers traités) pour tenir la qualité dans ses différentes dimensions. L'organisation elle-même devient alors "paradoxante » (op. cit.).

L'analyse de l'activité de gestion temporelle permet donc ici de renouveler l'approche des ajustements entre prescription stricte et réalité mouvante - question classique, mais prégnante dans une relation de service (Cerf \& Falzon, 2005 ; Creapt, 2017). Elle contribue en même temps à la prise en compte par l'organisation des dilemmes temporels qui en résultent, ceux-ci devant pouvoir être débattus et faire l'objet de réajustements, même provisoires, pour en atténuer les paradoxes.

\section{BIBLIOGRAPHIE}

Arnoud, J. \& Falzon, P. (2013). Changement organisationnel et reconception de l'organisation : des ressources aux capabilités. Activités, 10(2). https://journals.openedition.org/activites/760 Arnoud, J., Krohmer, C., \& Falzon, P. (2018). Dilemmes et paradoxes, quels effets sur le travail, quelles actions de prévention? Revue française de gestion, 274(5), 165-177.

Cellier, J.-M., De Keyser, V., \& Valot, C. (1996). La gestion du temps dans les environnements dynamiques. Paris : PUF.

Cerf, M., \& Falzon, P. (Eds.) (2005). Situation de service : travailler dans l'interaction. Paris : PUF.

Cloutier, E., David, H., Teiger, C., \& Prévost, J. (1999). Les compétences des auxiliaires familiales et sociales expérimentées dans la gestion des contraintes de temps et des risques de santé. Formation et Emploi, 67, 63-75.

CREAPT (2017). Travailler avec et pour un public : l'expérience des autres. Actes du séminaire Age et Travail [mai 2016]. Rapport de recherche du CEET, 104, pp. 119-138, Noisy le Grand : CEET. http:// 
www.cee-recherche.fr/sites/default/files/webfm/publications/rapportsderecherche/ 104_creapt_actes_sem_2016.pdf

Denis, J. (2011). Le travail de l'écrit en coulisses de la relation de service. Activités, 8(2), 32-52, https://journals.openedition.org/activites/2549.

Elias, N. (1993). Engagement et distanciation. Paris : Fayard.

Gaudart, C. (2014). Les relations entre l'âge et le travail comme problème temporel. Pistes, https://journals.openedition.org/pistes/

Grossin, W. (1996). Pour une science des temps. Introduction à l'écologie temporelle. Toulouse : Octarès.

Grossin, W. (2000). In quest of presents: how are they constructed? Social Science Information, 39, 657-663.

Jeantet, A. (2003). A votre service! La relation de service comme rapport social. Sociologie du Travail, 45(2), 91-209.

Joas, H. (2004, 1re éd. 1999). La créativité de l'agir (P. Rusch, Trans.). Paris : Édition du Cerf. Mead, G. H. (1967, 1st edt 1934). Mind, Self and Society from the Standpoint of a Social Behaviorist (Vol. 1). Chicago : University of Chicago Press.

Molinier, P. (2009) « Temps professionnel et temps personnel des travailleuses du care : perméabilité ou clivage ?». Temporalités, 9, http://journals.openedition.org/temporalites/988

Norros, L. (2004). Acting under uncertainty. The core task analysis in ecological study of work: Espoo 2004. VTT Publications 546.

Petit, J. (2005). Organiser la continuité du service : Intervention sur l'organisation d'une Mutuelle de santé . Université Bordeaux 2, ISPED, Laboratoire d'Ergonomie des Systèmes Complexes, Bordeaux.

Pueyo, V. (2012). Quand la gestion des risques est en péril chez les fondeurs. In A.-F. Molinié, C. Gaudart \& V. Pueyo (Eds.), La vie professionnelle : âge, expérience et santé à l'épreuve des conditions de travail (pp. 257-284). Toulouse : Octarès.

Simpson, B. (2013). Le pragmatisme, Mead et le tournant pratique. Activités, 10(1), https:// journals.openedition.org/activites/622

Teiger, C. (1987). L'organisation temporelle des activités. In C. Lévy-Leboyer \& J.-C. Sperandio (Eds.), Traité de psychologie du travail (pp. 659-687). Paris : PUF.

Volkoff, S. (2012). Le travail, en évolutions. In C. Gaudart, A.-F. Molinié \& V. Pueyo (Eds.), La vie professionnelle : âge, expérience et santé à l'épreuve des conditions de travail (pp. 31-42). Toulouse : Octarès.

Volkoff, S., Zara-Meylan, S., Mardon, C., \& Gaudart, C. (2016). Gérer les assurés de près et de loin. Un enjeu pour la qualité chez les Techniciens retraite. Connaissance de l'Emploi, Le Quatre pages du CEET $\mathrm{n}^{\circ}$ 131, Cnam. http://www.cee-recherche.fr/fr/connaissance_emploi.htm

Weill-Fassina, A., \& Pastré, P. (2004). Les compétences professionnelles et leur développement. In P. Falzon (Ed.), Ergonomie (pp. 213-232). Paris : PUF.

Zara-Meylan, V., 2013, Faire face aux imprévus sans être pris au dépourvu : le cas des chefs de culture dans de petites entreprises horticoles. Sociologies Pratiques, 26, 41-56. http:// www.cairn.info/revue-sociologies-pratiques.htm

Zara-Meylan, V. (2016). Quelles conceptions temporelles pour analyser l'activité ? Une proposition issue de recherches en ergonomie dans l'horticulture. Activités, 13(1), https:// journals.openedition.org/activites/2732. 
Zara-Meylan, V., \& Volkoff, S. (2017). Gérer les temps pour gérer la qualité : le traitement des dossiers retraite. $52^{e}$ Congrès de la SELF - Présent et Futur de l'Ergonomie (Toulouse, 20-22 septembre), pp 15-20. http://ergonomie-self.org/wp-content/uploads/2017/09/ActesSELF2017.pdf

Zara-Meylan, V., \& Volkoff, S, (2019). The dynamics of a service relationship: The example of technicians working for pension schemes. In S. Bagnara et al. (Eds.), IEA congress "Creativity in practice", Florence, August 2018, (Vol IV, pp. 310-319), Springer Nature.

\section{NOTES}

1. Date à laquelle est dû le premier versement d'une pension.

2. Ce logiciel propose notamment des fonctions « pilotage du portefeuille », « contacts " pour une trace des actions, le repérage de pièces manquantes ou d'incohérences de dates, et des alertes pour certains délais.

3. La mention TCC, TCR, TCo précise le métier du technicien Conseil (Carrières, Retraites ou Contrôle).

4. Les opérations effectuées par les techniciens carrières ne font pas l'objet d'un contrôle systématique.

5. Mutualité Sociale Agricole : autre régime auprès duquel l'assuré concerné disposait de droits.

6. Salaire annuel moyen.

\section{RÉSUMÉS}

L'objectif de cet article est d'examiner la gestion des cadres temporels multiples dans une activité de traitement de dossiers, dans ses liens avec la qualité. Nous analysons pour cela l'activité de techniciens retraite qui ont un rôle clé pour la fiabilité de la prestation de service public de l'organisme qui les emploie. À partir d'une méthodologie croisant observations de l'activité de back-office et entretiens à visée réflexive sur des dossiers en cours, nous analysons l'activité de gestion temporelle des techniciens dans leur activité quotidienne, puis plus transversalement dans des activités de régularisation de carrières, d'installation des rendez-vous et de gestion des « erreurs » retournées par le Contrôle. Nous montrons en quoi, avec l'expérience, les techniciens appréhendent les cadres temporels dans des configurations aux dynamiques multiples, qu'ils organisent et façonnent en cherchant à ouvrir des possibilités pour cette gestion. L'enjeu pour les systèmes de travail est alors de repérer différentes dimensions de la qualité du point de vue de l'activité individuelle et collective. Il s'agit en particulier d'identifier les besoins et enjeux de la gestion temporelle, ainsi que les configurations qui permettent la gestion des dilemmes temporels ou au contraire la rendent difficile.

This paper aims to analyze the relation between the management of multiple temporal frameworks in a case file processing activity and the quality of the service. This research is based on an analysis of the activity of "Pension Advice Technicians" in a French organization responsible for handling the case files of beneficiaries - future pensioners - and managing relations with them. In these situations, the quality of service is conventionally measured through an array of indicators used to steer the services provided and control the work carried 
out. However, these indicators do not measure the full range of skills used by members of staff. Our research aims to understand the activity of the technicians and address its temporal complexity in order to show how they ensure the quality of the service they provide in broader dimensions. Our methodology was based on an ergonomic analysis of the activity, adopting a holistic approach to the work and its challenges. It examines the dynamic management of their temporal frameworks, firstly within the working day and then more transversally in career regularization, appointment sets and management of "errors" returned by the Control. This profession, which requires high levels of administrative rigor, also requires an ability to manage the multiple dynamics involved, including the relationships the technicians establish with the beneficiaries themselves, as well as with the internal control bodies. From an ergonomics perspective, the aim is to ensure that the working methods do not ignore the need to construct and mobilize these temporal skills through experience, as this would be detrimental to the quality, but instead that they support their development. The issue of work system quality is then how to best support temporal framework management, taking into account the individual and collective activity and the resolution of temporal dilemmas inherent in the work.

\section{INDEX}

Mots-clés : analyse de l'activité, qualité, gestion temporelle, cadres temporels, dynamiques des systèmes de travail, organisation du travail et systèmes sociotechniques, techniques d'observation

Keywords : activity analysis, quality, dynamic processes, temporal management, temporal frameworks, accuracy and reliability, organizational design and management, observation methods

\section{AUTEURS}

\section{VALÉRIE ZARA-MEYLAN}

Cnam, Ceet et Gis Creapt, 29 promenade Michel Simon, 93166 Noisy le Grand, valerie.meylan@lecnam.net

\section{SERGE VOLKOFF}

Cnam, Ceet et Gis Creapt, 29 promenade Michel Simon, 93166 Noisy le Grand, serge.volkoff@lecnam.net 